\title{
Mini Review on Polycystic Ovary Syndrome (PCOS)
}

\section{Rajitha SR*}

Assistant Professor, Department of Obstetrics and Gynaecology Nursing, Christian College of Nursing, Neyyoor, The Tamilnadu Dr. MGR Medical University, Chennai, India

*Corresponding Author: Rajitha SR, Assistant Professor, Department of Obstetrics and Gynaecology Nursing, Christian College of Nursing, Neyyoor, The Tamilnadu Dr. MGR Medical University, Chennai, India.
Received: January 02, 2020

Published: February 10, 2020

(C) All rights are reserved by Rajitha SR.

DOI: 10.31080/ASWH.2020.02.0092

\begin{abstract}
PCOS is a serious genetic, metabolic, hormone and reproductive condition that commonly affects women and girls. It is an important cause of female infertility and a precursor for other critical conditions such as obesity, cardiovascular diseases, type - 2 diabetes and endometrial cancer. It has both reproductive and metabolic effects. However, PCOS is likely under diagnosed in most of the adolescent girls. It affects 5-10\% of all women of child-bearing age. Teenagers can experience the full range of PCOS symptoms seen in more mature women, including irregular or completely absent periods. No single cause has been identified; however, evidence suggests a complex interplay between genetic and environmental factors. It is very difficult to diagnose PCOS during the first 2 years following menarche because normal pubertal changes can mimic symptoms of PCOS. These symptoms are anovulatory menstrual cycles, transient multi-follicular ovarian morphology, increased androgen effects and relative insulin resistance. Common clinical features of PCOS include irregular or heavy or no menstrual periods, excess facial and body hair, acne, pain in the pelvis, difficulty to get pregnant and patches of thick, darker, velvety skin. Diagnosis of PCOS is based on any two of the following three findings. They are no ovulation, high androgen levels and ovarian cysts. Cysts may be distinguishable by ultrasonography. PCOS treatment may involve lifestyle modifications such as weight reduction and regular exercise. Birth control pills help to improve the regularity of menstrual periods, excess hair growth and acne. Metformin and anti-androgens may also help. Other typical acne treatments and hair removal techniques may be used for the treatment of PCOS. Weight loss, clomiphene or metformin also helps to improve fertility. The month of September is considered as Polycystic Ovary Syndrome (PCOS) awareness month around the world. It helps to initiate a conversation about this under diagnosed medical condition. Women who have PCOS are at high risk for several serious complications, some of them can be life threatening complications. Early diagnosis and treatment helps to reduce the risk for many of the complications. Keywords: Hyperinsulinemia; Hirsutism; Anovulation; Electrocautery; Hyperandrogenemia
\end{abstract}

\section{Introduction}

Polycystic Ovary Syndrome (PCOS) is a hormonal disorder common among women of reproductive age. Infrequent or prolonged menstrual periods or excess male hormone (androgen) levels can be seen in PCOS women. The ovaries may develop numerous tiny in size collections of fluid (follicles) and fail to release the eggs regularly [1]. PCOS was first described by American gynecologists Irving F. Stein, Sr. and Michael L. Leventhal in 1935, from whom its original name of Stein-Leventhal syndrome is taken. The primitive published description of a person with what is now recognized as PCOS was in Italy in the year of 1721 and in 1844 cyst-related changes to the ovaries were described [2]. Alternative names of PCOS include polycystic ovaries, polycystic ovary disease, polycystic ovarian disease, Stein-Leventhal syndrome, polyfollicular ovarian disease [3], cystic disease of ovary, multicystic ovaries, sclerocystic ovarian degeneration, sclerocystic ovaries, sclerocystic ovary syndrome [4] and functional ovarian hyperandrogenism [2].

\section{Incidence}

The World Health Organization (WHO) estimates that, PCOS affected with 116 million women (3.4\%) worldwide in 2012. Globally, the prevalence of PCOS is highly variable, that ranges from $2.2 \%$ to as high as $26 \%$ [5]. The growing trend of PCOS is predominantly seen in the age group of 15 to 30 years [6]. One in every 10 women has PCOS in India, a common endocrinal system disorder among women of reproductive age, according to a study by the PCOS Society and out of every 10 women diagnosed with PCOS, six are teenage girls [7].

\section{Etiology and risk factors}

The exact causes of PCOS are uncertain, but the factors that are most likely are: hormonal imbalance - This occurs as ovaries produce androgens (male hormones) more than normal. These high levels of androgens affect the development and release of the eggs during ovulation; insulin excess - Insulin is the hormone that al- 
lows the cell to use up glucose and convert it into energy. When there is insulin resistance, the ability to use insulin effectively is affected and this causes excess secretion of insulin to combat the resistance. This excess boosts the ovaries to produce more androgens and contributes as one of the causes of PCOS; heredity - The likelihood of familial predisposition to PCOS is more if the disease is known to run in the family [8]; low-grade inflammation - This term is used to describe white blood cells' production of substances to fight infection. Low-grade inflammation stimulates polycystic ovaries to produce androgens, which can lead to heart and blood vessel problems; and excess androgen - The ovaries produce abnormally high levels of androgen, resulting in hirsutism and acne [1].

\section{Pathophysiology}

There are several disease mechanisms at work in PCOS, but one of the most significant involves insulin. Hyperinsulinemia has been identified as one of the underlying causes of many of the symptoms of PCOS. Too much insulin in the body alters the ovaries' ability to convert androstenedione into estrone and estradiol and enhances the tendency of the theca cells of the ovary to convert more of this to testosterone, instead. The theca cells in affected women are more efficient at converting androstenedione into testosterone than estradiol in the first place.

The presence of testosterone typically stimulates the liver to produce sex-hormone-binding-globulin (SHBG) to bind to the testosterone and neutralize it, but insulin suppresses the synthesis of SHBG, which leads to elevated levels of free testosterone in the blood, resulting in hyperandrogenemia. Excess insulin floating around in the circulation of the body may also lead to insulin resistance, as the body's tissues get more accustomed to excess insulin being produced. If this is not treated, in time this can result in impaired glucose tolerance, and late, to Type II Diabetes Mellitus. The body also uses insulin to convert energy into fat, and too much insulin in the body can make it more difficult for women with PCOS to manage their weight. "Hyperinsulinemia also produces the pituitary to hypersecrete luteinizing hormone (LH), which activates anovulation". Anovulation is the cause of the majority of the infertility experienced by women with PCOS [9].

\section{Signs and Symptoms}

Some common signs and symptoms of PCOS include acne, darkening of skin, hirsutism (abnormal growth of hair on face and body), irregular menstrual cycle, skin tags, thinning hair, weight gain [10], oily skin, infertility or repeat miscarriages, depression and/or mood swings [11], darkened skin patches, anxiety, sleep apnea [12], swelling in legs or feet, migraines or headaches, body pain, fatigue, migraines or headaches, moon face, buffalo hump, painful intercourse and breathlessness or wheezing [13].

\section{Diagnosis}

Medical history - it includes the menstrual periods and weight changes; a physical examination - it includes checking for signs of excess hair growth, acne and insulin resistance [1] (a waistline over 35 inches in women, blood pressure of 130/80 or higher, a fasting glucose level over $100 \mathrm{mg} / \mathrm{dL}$, a fasting triglyceride level over $150 \mathrm{mg} / \mathrm{dL}$, a HDL cholesterol level over under $50 \mathrm{mg} / \mathrm{dL}$ in women, skin tags and patches of dark, velvety skin called acanthosis nigricans) [14]; a pelvic examination - the doctor visually and manually inspects the reproductive organs for masses, growths or other abnormalities; blood tests - blood may be analyzed to measure the hormone levels [1]. It includes, Follicle Stimulating Hormone (FSH) - it affects the ability to get pregnant [15]. The normal FSH level in menstruating women is about $4.7-21.5 \mathrm{mIU} / \mathrm{ml}$ [16]. It might be lower than normal, or even normal in PCOS. Luteinizing Hormone (LH) - it encourages ovulation and it could be higher than normal in PCOS. The LH levels can vary at different times of the menstrual cycle: at the follicular phase of the menstrual cycle: 1.9 to $12.5 \mathrm{IU} / \mathrm{L}$; at the peak of the menstrual cycle: 8.7 to $76.3 \mathrm{IU} / \mathrm{L}$ and during the luteal phase of the menstrual cycle: 0.5 to $16.9 \mathrm{IU} / \mathrm{L}$ [17]. Testosterone - it is a sex hormone and it would be higher in PCOS. The normal testosterone level in women is about 15-70 $\mathrm{ng} / \mathrm{dL}$ [18]. Estrogens - These are group of hormones that allows women to get their periods and it may be normal or high in PCOS. The normal range of estrogen varies depending upon the woman's age: 20 - 29 years - $149 \mathrm{pg} / \mathrm{ml} ; 30$ to 39 years - $210 \mathrm{pg} / \mathrm{ml}$; and over 40 years but not in menopause - 152 pg/ml [19]. Sex Hormone Binding Globulin (SHBG) may be lower than normal. The normal level of SHBG is about 18-144 nmol/L [20]. Androstenedione - it is a sex hormone and it may be at a higher than normal level [14]. The normal range of androstenedione is about $30-200 \mathrm{ng} / \mathrm{dL}$ in females [21]. Blood testing can exclude the possible causes of menstrual irregularities or excessive androgen that mimics PCOS. In addition, blood testing also helps to measure the fasting cholesterol, glucose tolerance and triglyceride levels. Serum (blood) levels of androgens (hormones associated with male development), including androstenedione and testosterone may be elevated; ultrasonography - it shows the appearance of ovaries and the thickness of the lining of the uterus [1]; laparoscopic examination - it may reveal a thickened, smooth, pearl-white outer surface of the ovary [2]; the guidelines from the Endocrine Society recommend using the Rotterdam criteria for the diagnosis of PCOS. The Rotterdam criteria mandate the presence of at least two of the following three findings: hyperandrogenism - either clinically by skin manifestations of androgen excess or hyperandrogenemia (high testosterone in a blood test), ovulation dysfunction (oligo/anovulation), and polycystic ovaries on ultrasound [22]; and NIH (National Institutes of Health)/NICHD (National Institutes of Child Health and Human Development) criteria requires the presence of both following features: oligo and/ anovulation and clinical or biochemical hyperandrogenism [23]. 


\section{Differential diagnoses}

Differential diagnoses of PCOS include Congenital Adrenal Hyperplasia (CAH), Cushing's syndrome, ovarian hyperthecosis, hypothyroidism [24,25], pregnancy, androgen producing tumors [26], and other pituitary or adrenal disorders [2].

\section{Management of PCOS}

Lifestyle modifications

A right diet with adequate exercise has shown great results in PCOS recovery. Diet should be dominated with lots of fruits and vegetables on a daily basis. Minimum of 2 - 3 liters of water/day is essential. Regular exercise for 30 - 40 minutes daily (Brisk walking, yoga, and meditation) is recommended [15]. Obese women with PCOS can benefit from a low-calorie diet for their weight reduction [27].

\section{Medical management}

There are various number of medical therapies used to reduce the different symptoms of PCOS. It includes for periods, fertility, excess hair, acne and weight gain. The medical therapies are oral contraceptive pills, insulin sensitizing drugs such as Metformin, hormones (gonadotrophins), testosterone lowering drugs, weight losing drugs, antidepressants and anti-anxiety drugs [27].

\section{Surgical management}

Surgical management of PCOS is aimed mainly at restoration of ovulation. Ovarian wedge resection has been advocated because of postoperative adhesion formation and the successful administration of ovulation-inducing medications. Various laparoscopic methods, including electrocautery, laser drilling and multiple biopsies have been used with the aim of creating certain focal areas of damage in the ovarian cortex and its stroma [16].

\section{Complications}

The complications of PCOS include: diabetes [28] (insulin resistance by the receptors for insulin leads to high levels of insulin being produced by the pancreas) [29], obesity [28] (hyperandrogenemia affects the distribution of body fat) [30] infertility [28], (due to the development of numerous fluid-filled sacs in the ovaries and failure of ovaries to regularly release eggs for reproduction, hormonal imbalances and any abnormality in the menstrual cycle may also interfere with ovulation) [31] atherosclerosis (clogged arteries), high blood pressure (hypertension), heart attack [32] (increased levels of androgens and the low level of adiponectin cause cardiac diseases) [33], breast cancer [32], endometrial cancer (prolonged ovarian function disorder in women with polycystic ovarian syndrome can alter endometrial cell growth, which in turn can cause cancer), metabolic syndrome [34]. (central obesity, hypertension, atherogenic dyslipidemia, and insulin resistance in PCOS cause metabolic syndrome) [35] and sleep apnea [34] (insulin resistance makes difficult for the body to absorb energy, which can cause weight gain and that can lead to trouble sleeping soundly) [36].

\section{Prognosis}

Women who have polycystic ovary syndrome are at increased risk for serious complications, some of which can be life threatening. Early diagnosis and treatment can help reduce the risk for many of the complications. Women who have PCOS should receive regular medical care (including screening for diabetes, high blood pressure, and high cholesterol) and should maintain a healthy lifestyle by eating right and exercising regularly. Women who have PCOS should not smoke [37] because smoking is harmful for health and it increases the risks of PCOS-related cardiovascular diseases such as hypertension, atherosclerosis, and heart attack [38].

\section{Conclusion}

Due to a combination of genetic and environmental factors, PCOS occurs. Risk factors of PCOS are obesity, a lack of physical exercise and a family history of PCOS. Diagnosis of PCOS is based on any two of the following three findings. They are no ovulation, high androgen levels and ovarian cysts. Cysts may be distinguishable by ultrasonography. Differential diagnoses of PCOS include adrenal hyperplasia, Cushing's syndrome, pregnancy, hypothyroidism and high blood levels of prolactin. PCOS treatment may involve lifestyle modifications such as weight reduction and regular exercise. The medical therapies are oral contraceptive pill, insulin sensitizing drugs such as Metformin, hormones (gonadotrophins), testosterone lowering drugs, weight loss drugs, antidepressants and antianxiety drugs. Early diagnosis and treatment can help reduce the risk for many of the complications.

\section{Bibliography}

1. Polycystic Ovary Syndrome (PCOS). Mayo Clinic (2019).

2. Polycystic Ovary Syndrome. From Wikipedia, the free encyclopedia (2019).

3. Polycystic Ovary Syndrome. Medline Plus (2019).

4. Polycystic Ovary Syndrome. US National Library of Medicine (2019).

5. Usha B., et al. "An epidemiological survey: Effect of predisposing factors for PCOS in Indian urban and rural population". Middle East Fertility Society Journal 22.4 (2017): 313-316.

6. Sobiya NM. " 1 in 5 women affected by PCOS in India! But fret not, we have the solution". Health (2015).

7. Sunitha PS. "Why PCOS on the rise among Indian women" (2017). 
8. PCOS (Polycystic Ovary Syndrome) Causes (2019).

9. Danette T. "Polycystic Ovary Syndrome: An Overview” (2018).

10. Polycystic Ovary Syndrome - PCOS or PCOD (2019).

11. Tandy GO. "Signs and Symptoms of Polycystic Ovary Syndrome". Intermountain Health Care (2018).

12. Symptoms and Causes. Jeans Hailes for Women's Health (2019).

13. 30 PCOS symptoms you don't want to ignore (2018).

14. Insulin Resistance. WebMD (2019).

15. PCOD. Life Force Homeopathy (2018).

16. What is a Normal FSH Level? Reference (2020).

17. Luteinizing Hormone (LH) Test - Procedure, Preparation and Normal Range (2018).

18. What are Normal Testosterone Levels in Women? (2017).

19. Range of Normal Estrogen Levels. Healthfully (2017).

20. Sex Hormone-Binding Globulin (SHBG), Serum. Mayo Foundation for Medical Education and Research (2019).

21. Androstenedione, Serum. Foundation for Medical Education and Research (2019).

22. Abdallah M. "Rotterdam Criteria for diagnosing Polycystic Ovarian Syndrome (PCOS)". Time of Care Online Medicine Notebook (2016).

23. Sirmans SM and Pate KA. "Epidemiology, diagnosis, and management of polycystic ovary syndrome". Clinical Epidemiology 6 (2014): 1-13.

24. Sari C. "Differential Diagnosis of PCOS” (2018).

25. Richard SL. Polycystic Ovarian Syndrome Differential Diagnoses. MedScape (2019).

26. Jason F. “Differential Diagnosis of PCOS” (2018).

27. Management and treatment. Jeans Hailes for Women's Health (2019).

28. Complications of Polycystic ovary syndrome. Right Diagnosis (2015)

29. Krans B. "What's the Connection between Polycystic Ovarian Syndrome (PCOS) and Diabetes?" Healthline (2018).
30. PCOS and Obesity (2018).

31. PCOS and the Role it plays in Women's Infertility (2018).

32. Polycystic Ovary Syndrome (PCOS): Symptoms, Complications and Treatment (2018).

33. Green E. "What is PCOS? Causes, Symptoms and Treatment?" Pregworld (2017).

34. Complications. Jean Hailes for Women's Health (2019).

35. Polycystic Ovary Syndrome can be the main cause of Metabolic Syndrome (2019).

36. Nielsen R. "PCOS and Sleep Apnea: Is There a Link?". Health Status (2018).

37. Polycystic Ovary Syndrome (2015).

38. Fermi S. "Things not to do during PCOS” (2019).

\section{Assets from publication with us}

- Prompt Acknowledgement after receiving the article

- Thorough Double blinded peer review

- Rapid Publication

- Issue of Publication Certificate

- High visibility of your Published work

Website: https://www.actascientific.com/

Submit Article: https://www.actascientific.com/submission.php Email us: editor@actascientific.com

Contact us: +919182824667 